\title{
Survey Paper on Digital Image Sharing by Diverse Image Media
}

\author{
Ashwini Udmale \\ Department of Computer Engineering \\ Sinhgad Institute of Technology, Lonavala \\ Pune, Maharashtra
}

\author{
S.B. Nimbekar \\ Department of Computer Engineering \\ Sinhgad Institute of Technology, Lonavala \\ Pune, Maharashtra
}

\begin{abstract}
Conventional cryptography is a method of transforming message into an unreadable format called as cipher text. This cipher text can easily be shared over the network without fear but anyone can get message easily by applying key on cipher text. Sharing and delivering secret images over the network is also known as a visual secret sharing (VSS) scheme. However main drawback of VSS scheme is that it is experience from high transmission risk due to the shares are like noise. As the shares are like noise which in turn lead to cause the attackers attention. To solve this problem mechanism is developed called Natural image based visual secret sharing (NVSS).

This paper is motivated by need of novel mechanism to transfer the secret images over network. In proposed work for regeneration of secret image a generated noisy share, natural images and seed (needed for random functions used) are required at the receiving end. Though generated share is noisy and its contents are not recognizable, overall structure may be visible in some cases. So data hiding is should be performed on generated share to hide that structure (texture) in noisy share.
\end{abstract}

\section{Keywords}

Visual Secret Sharing Scheme: Natural image; Encryption; Steganography.

\section{INTRODUCTION}

Tremendous growth of Internet which is the group of computer and communicating devices that are connected together through some wired or wireless media to transmit various range of information and services demands better security. Traditional Cryptography is a process of converting the original data into an unreadable encrypted format called the cipher text. Cryptography uses the hash function that in turn uses mathematical function to encrypt the data which protects the data during transmission and to transmit confidential data over the communication media.

Traditional shares, which contain many random and meaningless pixels, although they satisfy the security requirement for protecting secret contents but they suffer from two major drawbacks such as a high transmission risk because holding noise-like shares will cause attract attacker attention and second the meaningless shares are not user friendly. If the number of shares of image enhanced, it becomes trickier to retain the shares, which never give any information for identifying the shares.

A method that encrypts a secret image into $\mathrm{n}$ shares, with each participant holding one or more shares is called Visual cryptography (VC). Secret images can be in the form of images, handwritten documents, photographs and so on. Sharing and delivering secret images over the network is also known as a visual secret sharing (VSS) scheme. Visual secret sharing scheme (VSS) is the process of sharing of image securely in a non computer environment [1].

\section{RELATED WORK}

P. L. Chiu and K. H. Lee proposed a pixel-expansion-free threshold VCSs approach on the basis of optimization technique to encrypt binary secret images. For the evaluation of the display quality of recovered images author consider blackness as one of the performance metric. Their work is consider as two-fold approach first formulate the problem as a mathematical optimization model in turn to maximize the contrast of recovered images that are lead to density-balance and blackness constraints and second they develop a simulated-annealing-based algorithm to solve this problem. The proposed optimization-based approach significantly outperforms previous methods in terms of both the pixel expansion factor and the display quality of recovered images[2].

K. H. Lee and P. L. Chiu proposed visual cryptography experiences a pixel-expansion problem, or an uncontrollable display quality issues for recovered images. To address these issues in paper, author proposed a general and systematic approach without sophisticated codebook design. Proposed approach can be used for binary secret images in non-computer-aided decryption environments. To encrypt secret pixels author design a set of column vectors rather than using the conventional VC-based approach to avoid pixel expansion[3].

Z. Wang, G. R. Arce, and G. D. Crescenzo proposed a general framework called halftone visual cryptography to achieve visual cryptography via halftoning. On the basis of blue-noise dithering principles, the proposed method make use of the void and cluster algorithm to encode a secret binary image into halftone shares carrying significant visual information. The achieved visual quality is better than all previous visual cryptography method. The proposed method has a number of visual secret sharing applications such as watermarking, electronic cash which require high-quality visual images[4].

I. Kang, G. R. Arce, and H. K. Lee proposed mechanism called a color visual cryptography encryption which generates meaningful color shares via VIP synchronization and error diffusion for visual quality improvement. Visual information pixel (VIP) synchronization maintain the same original VIP values before and after encryption and error diffusion generates shares with high visual quality. VIP synchronization or error diffusion mostly used in various visual cryptography schemes for color images. As compare with previous approaches proposed approach gives superior performance[5]. 
T. H. N. Le, C. C. Lin, C. C. Chang, and H. B. Le proposed a new secret sharing scheme for grayscale images. Proposed scheme is based on three approaches, block truncation coding (BTC), discrete wavelet transform (DWT) and vector quantization (VQ) technique. An original image is replaced with a set of much smaller shadows and each shadow does not reveal information about the original image. Due to this quality the security of the proposed scheme is guaranteed. This proposed scheme can be applied to both grayscale and color images. Results confirm that this scheme not only generates a high quality reconstructed original image but also generates small, random-like grayscale shadows[6].

D. S. Tsai, G. Horng, T. H. Chen, and Y. T. Huang proposed a novel and efficient secret image sharing scheme for true-color secret images. By combing neural networks and variant visual secret sharing, the quality of the reconstructed secret image and disguise images are visually the same as the original images. Only proposed scheme supports true-color secret image with size constraint on shares as compare to other[7].

D. S. Tsai, G. Horng, T. H. Chen, and Y. T. Huang proposed anew secret image sharing scheme with reversible steganography. A reversible cellular automaton with memory is added in the proposed scheme to produce shared data, which are implanted into cover image to form stego images. Computation cost of the proposed scheme is lower than other approaches. The proposed scheme is useless in differential attacks[8]

\section{Table 1: Survey Table}

\begin{tabular}{|c|c|c|c|}
\hline Paper & Prepositions & Advantages & Disadvantages \\
\hline $\begin{array}{l}\text { 1] K. H. Lee and P. L. Chiu, "Image } \\
\text { size invariant visual cryptography } \\
\text { for } \\
\text { general access structures subject to } \\
\text { display quality constraints," IEEE } \\
\text { Trans. Image Process., vol. } 22 \text {, no. } \\
10 \text {, pp. } 3830-3841 \text {, Oct. } 2013\end{array}$ & $\begin{array}{l}\text { 1. Author proposed a general } \\
\text { and systematic approach } \\
\text { without sophisticated } \\
\text { codebook design. }\end{array}$ & $\begin{array}{l}\text { 1. Proposed approach can } \\
\text { be used for binary secret } \\
\text { images in non-computer- } \\
\text { aided decryption } \\
\text { environments. }\end{array}$ & $\begin{array}{lr}\text { 1. Suffered } & \text { from } \\
\text { transmission } & \text { risk } \\
\text { problem. } & \end{array}$ \\
\hline $\begin{array}{l}\text { 2] Kai-Hui Lee and Pei-Ling Chiu, } \\
\text { "An Extended Visual Cryptography } \\
\text { Algorithm for General Access } \\
\text { Structures ," in Proc. IEEE } \\
\text { TRANSACTIONS } \\
\text { INFORMATION FORENSICS AND } \\
\text { SECURITY, VOL. 7, NO. } 1 \text {, } \\
\text { FEBRUARY 2012 }\end{array}$ & $\begin{array}{l}\text { 1. Author proposed a Solve } \\
\text { the share identification } \\
\text { problem using extended } \\
\text { visual cryptography (EVCS), } \\
\text { which adds a meaningful } \\
\text { cover image in each share. }\end{array}$ & $\begin{array}{l}\text { 1. Reduce the pixel } \\
\text { expansion problem. } \\
\text { 2. Better performances }\end{array}$ & $\begin{array}{l}\text { 1. Work only black and } \\
\text { white images. } \\
\text { 2. Low quality display. }\end{array}$ \\
\hline $\begin{array}{l}\text { 3] F. Liu and C. Wu,"Embedded } \\
\text { extended visual cryptography } \\
\text { schemes", IEEE Trans. Inf. Forensics } \\
\text { Security, vol. 6, no. 2, pp. 307-322, } \\
\text { Jun. } \\
\text { 2011. }\end{array}$ & $\begin{array}{l}\text { 1.In this paper propose a } \\
\text { construction of EVCs which } \\
\text { is realized by embedding } \\
\text { random shares into } \\
\text { meaningful covering shares } \\
\text { and author call it the } \\
\text { embedded EVCs. } \\
\text { 2. Author also proposed a } \\
\text { method to improve the visual } \\
\text { quality of the share images. }\end{array}$ & $\begin{array}{l}\text { 1. Better visual quality } \\
\text { more than others EVCs. }\end{array}$ & 1. Not user friendly. \\
\hline $\begin{array}{l}\text { 4] R. Z.Wang, Y. C. Lan, Y. K. Lee, } \\
\text { S. Y. Huang, S. J. Shyu, and T. L. } \\
\text { Chia, } \\
\text { "Incrementing visual cryptography } \\
\text { using random grids," Opt. Commun., } \\
\text { vol. } 283 \text {, no. 21, pp. 4242-4249, Nov. } \\
2010 \text {. }\end{array}$ & $\begin{array}{l}\text { 1. Focuses on using } \\
\text { transparencies or digital } \\
\text { media as carriers for a VSS } \\
\text { scheme. } \\
\text { 2. The transparency shares } \\
\text { have either a noise-like or a } \\
\text { meaningful appearance. }\end{array}$ & $\begin{array}{l}\text { 1. No pixel expansion } \\
\text { during encryption or } \\
\text { decryption. } \\
\text { 1. Codebook is used for } \\
\text { encryption methods }\end{array}$ & $\begin{array}{l}\text { 1.Communication cost } \\
\text { is more }\end{array}$ \\
\hline $\begin{array}{l}\text { 5] Z. Wang, G. R. Arce, and G. D. } \\
\text { Crescenzo, "Halftone visual } \\
\text { cryptography } \\
\text { via error diffusion," IEEE Trans. Inf. } \\
\text { Forensics Security, vol. 4, } \\
\text { no. 3, pp. 383-396, Sep. } 2009 \text {. }\end{array}$ & $\begin{array}{l}\text { 1. This is used for error } \\
\text { diffusion process. } \\
\text { 2. Error diffusion has low } \\
\text { complexity but support } \\
\text { halftone share images to good } \\
\text { extent of image quality. }\end{array}$ & $\begin{array}{lcr}\text { 1.High } & \text { quality } & \text { visual } \\
\text { images such as } & \text { no. of } \\
\text { visual secret } & \text { sharing } \\
\text { applications } & \text { as } \\
\text { watermarking, electronics } \\
\text { cash etc. }\end{array}$ & 1. Low complexity \\
\hline
\end{tabular}


3. ARCHITECTURAL VIEW

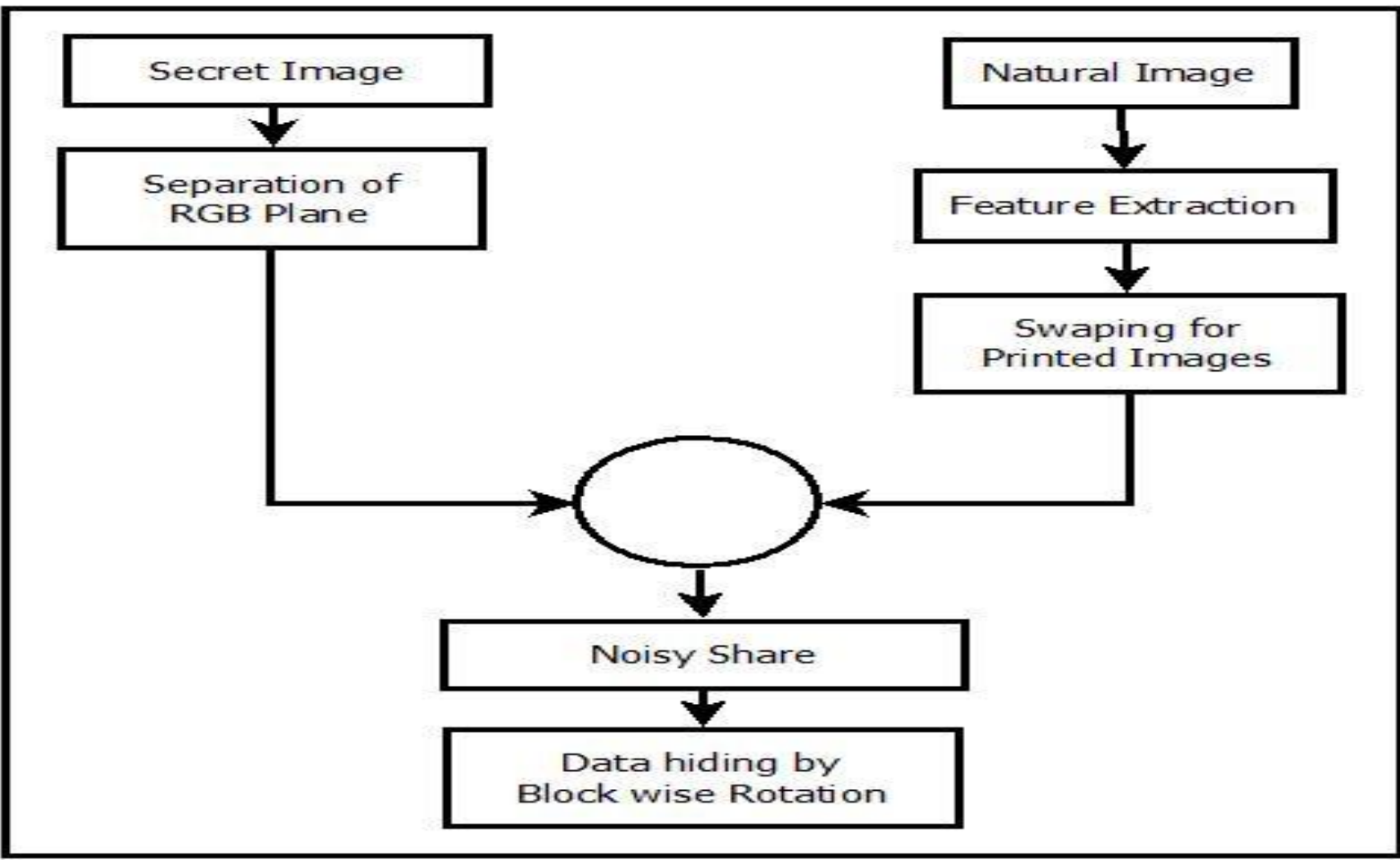

Figure 2: Encryption Process

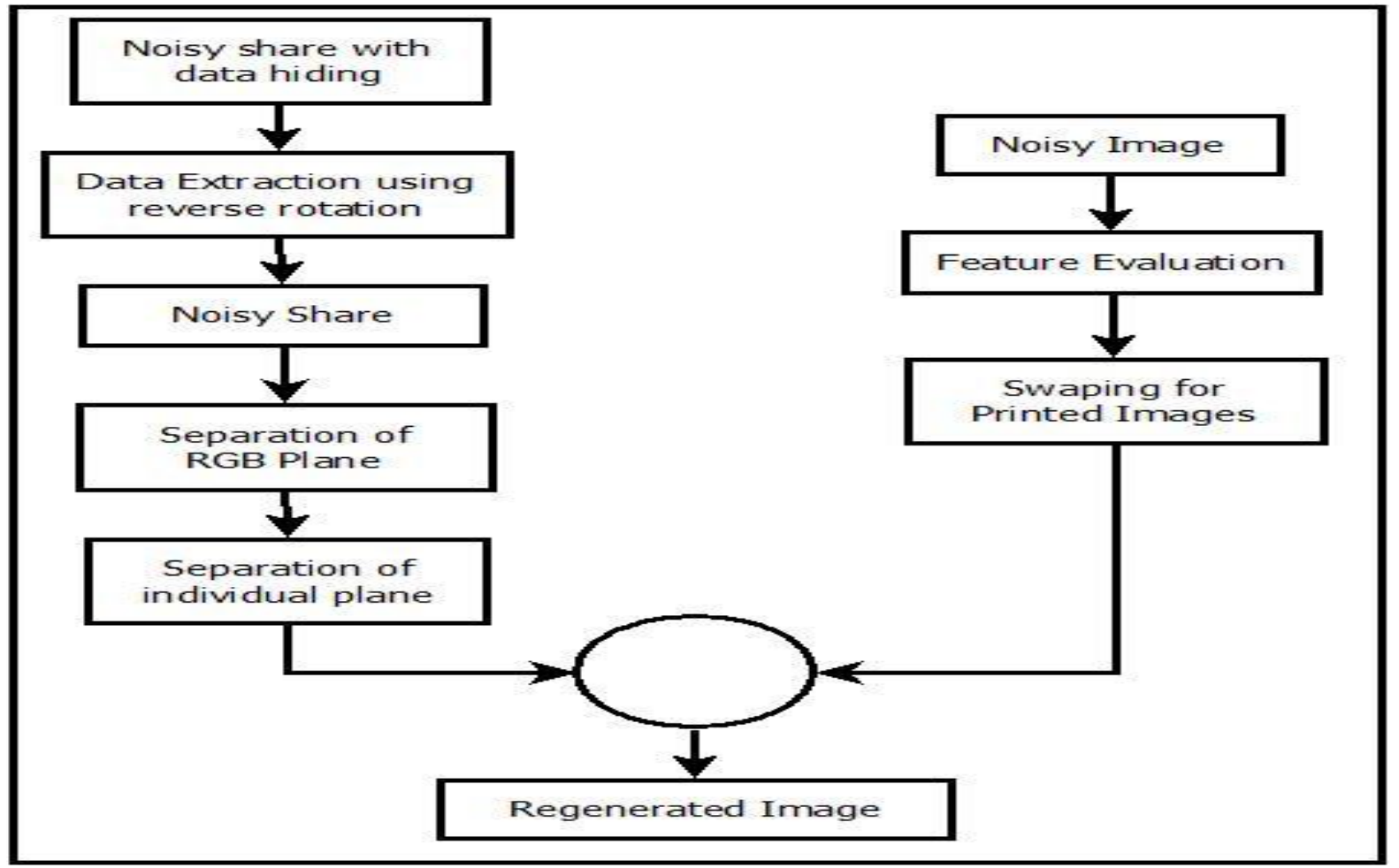

Figure 3: Decryption Process

\section{CONCLUSION AND FUTURE SCOPE}

This paper presented an all-inclusive survey of secret sharing scheme. The main features, the advantages and disadvantages of each are described. As per survey, strong need to develop the secure secrete scheme for sharing images over network.
In proposed work is the combination of data hiding, blockwise rotation and the encryption technique. Using this we achieve the original image with security. 


\section{ACKNOWLEDGMENTS}

The authors would like to thank the researchers as well as publishers for making their resources available and teachers for their guidance.

\section{REFERENCES}

[1] Kai-Hui Lee and Pei-Ling Chiu,"Digital Image Sharing by Diverse Image Media", IEEE TRANSACTIONS ON INFORMATION FORENSICS AND SECURITY, VOL. 9, NO. 1, JANUARY 2014.

[2] P. L. Chiu and K. H. Lee, "A simulated annealing algorithm for general threshold visual cryptography schemes," IEEE Trans. Inf. Forensics Security, vol. 6, no. 3, pp. 992-1001, Sep. 2011.

[3] K. H. Lee and P. L. Chiu, "Image size invariant visual cryptography for general access structures subject to display quality constraints," IEEE Trans. Image Process., vol. 22, no. 10, pp. 3830-3841, Oct. 2013.

[4] Z. Wang, G. R. Arce, and G. D. Crescenzo, "Halftone visual cryptography via error diffusion," IEEE Trans. Inf. Forensics Security, vol. 4, no. 3, pp. 383-396, Sep. 2009.

[5] I. Kang, G. R. Arce, and H. K. Lee, "Color extended visual cryptography using error diffusion," IEEE Trans. Image Process., vol. 20, no. 1, pp. 132-145, Jan. 2011.
[6] T. H. N. Le, C. C. Lin, C. C. Chang, and H. B. Le, “A high quality and small shadow size visual secret sharing scheme based on hybrid strategy for grayscale images," Digit. Signal Process., vol. 21, no. 6, pp. 734-745, Dec. 2011.

[7] D. S. Tsai, G. Horng, T. H. Chen, and Y. T. Huang, "A novel secret image sharing scheme for true-color images with size constraint," Inf. Sci., vol. 179, no. 19, pp. 3247-3254, Sep. 2009.

[8] X. Wu, D. Ou, Q. Liang, and W. Sun, "A user-friendly secret image sharing scheme with reversible steganography based on cellular automata," J. Syst. Softw., vol. 85, no. 8, pp. 1852-1863, Aug. 2012.

[9] C. Guo, C. C. Chang, and C. Qin, "A multi-threshold secret image sharing scheme based on MSP," Pattern Recognit. Lett., vol. 33, no. 12, pp. 1594-1600, Sep. 2012.

[10] P. L. Chiu, K. H. Lee, K. W. Peng, and S. Y. Cheng, "A new color image sharing scheme with natural shadows," in Proc. 10th WCICA, Beijing, China, Jul. 2012, pp. 4568-4573. 